\section{CASE REPORT}

\author{
S.A. $\mathrm{Ng}$ \\ I. Corcuera-Solano \\ V.V. Gurudutt \\ P.M. Som
}

\title{
A Rare Case of Reidel Thyroiditis with Associated Vocal Cord Paralysis: CT and MR Imaging Features
}

\begin{abstract}
SUMMARY: Reidel thyroiditis is extremely rare and not only involves the thyroid gland but usually extends to neighboring structures in the neck. A rare complication of this disease is entrapment of the recurrent laryngeal nerve causing a vocal cord paralysis. In fact, to our knowledge, this is likely the only benign thyroid disease to cause such a paralysis. We present a case of a 57-year-old woman with Reidel thyroiditis and a recurrent laryngeal nerve paralysis. The CT and MR imaging features are presented as well as a brief review of this disease.
\end{abstract}

$\mathbf{R}$ eidel thyroiditis is the rarest form of thyroiditis, accounting for less than $0.06 \%$ of patients undergoing thyroid biopsies in 1 series and $0.06 \%$ of the thyroidectomy cases in another large series. ${ }^{1-4}$ There is a female predominance, and the diagnosis is made between 30 and 60 years of age.$^{5-7}$ It can not only involve the thyroid gland but also extend out into the adjacent neck structures, rarely infiltrating the recurrent laryngeal nerve. In a literature review, it is the only benign thyroid disease to cause recurrent laryngeal nerve palsy.

\section{Case Report}

The patient was a 57-year-old woman with a 10-month history of thyroid mass. She had had multiple fine-needle sonographic biopsies and an open biopsy, all of which were consistent with Reidel thyroiditis. Three months earlier, she noted dysphagia and hoarseness, and endoscopy had revealed a right recurrent laryngeal nerve paralysis. She was started on tamoxifen therapy with no significant improvement after 2 months. On physical examination, she had a very firm low-neck fixed mass, which was nontender and not painful to palpation. A CT scan showed a large highly infiltrating thyroid mass that primarily involved the right thyroid lobe and isthmus but also extended into the left thyroid lobe. Within the gland, there were several high-attenuation nodules, some with small calcifications. The extraglandular disease laterally displaced and encased the right common carotid artery, narrowing its lumen. There was encasement of the trachea with an approximately $20 \%$ narrowing of the airway. The fat planes about the cervical esophagus and the flexor cervicalis muscles were also infiltrated. The mass extended through the cricothyroid membrane, and there was laryngeal ventricular dilation, medialization of the aryepiglottic fold, and fullness of the true cord, all consistent with the clinical diagnosis of a right vocal cord paralysis.

MR imaging (Fig 1) confirmed the overall morphology of the mass seen on CT. There was a low T1-weighted signal intensity and a lower T2-weighted signal intensity with a 5-cm higher signal-intensity central mass, which had areas of necrosis. This central mass enhanced more than the surrounding attenuated fibrous scar. This internal mass was not well-appreciated on CT. There were no pathologic nodes seen in the neck. The patient was taken to the operating room

Received August 19, 2010; accepted after revision September 19.

From the Departments of Radiology (S.A.N., P.M.S.) and Otolaryngology (V.V.G.), Mount Sinai School of Medicine of New York University, New York, New York; and Hospital de la Santa Creu I Sant Pau (I.C.-S.), Barcelona, Spain.

Please address correspondence to Peter Som, MD, FACR. Department of Radiology, The Mount Sinai Medical Center, One Gustave Levy PI, New York, NY 10029; e-mail: peter.som@mssm.edu

http://dx.doi.org/10.3174/ajnr.A2406 where the right thyroid lobe was removed and as much debulking of the thyroid mass as possible was performed. Pathology revealed attenuated fibrous tissue with pockets of benign thyroid tissue with focal oncocytic metaplasia consistent with Reidel thyroiditis. Postoperatively, her dysphagia has improved and her voice is stronger.

\section{Discussion}

Reidel thyroiditis, also known as Reidel struma and invasive fibrous thyroiditis, was first described by Bernhard Reidel in 1896. ${ }^{8,9}$ Multiple hypotheses regarding its pathogenesis have been postulated; however, the exact etiology remains uncertain. Of these hypotheses, an autoimmune etiology currently seems most likely. ${ }^{1,10}$ To our knowledge, there have been no case reports of distant metastasis, though there are rare reports of a concomitant thyroid malignancy. ${ }^{11}$

Pathologically, this disease is secondary to a chronic inflammatory reaction of the normal thyroid parenchyma. ${ }^{12}$ As the inflammation progresses, the gland is infiltrated by lymphoid and plasma cells and variable numbers of eosinophils. ${ }^{4,8,10}$ Gradually the gland is replaced by attenuated hyalinized fibrous tissue. ${ }^{8}$ Unlike the fibrosing variant of Hashimoto thyroiditis, which remains confined to the thyroid gland, the attenuated fibrous reaction of Reidel thyroiditis extends beyond the thyroid capsule to engulf adjacent structures, leading to vascular, tracheal, and/or esophageal compression as well as involvement of the parathyroid glands, causing hypoparathyroidism. ${ }^{5-8,10}$ Involvement of the recurrent laryngeal nerve can rarely occur, resulting in a vocal cord paralysis. ${ }^{8,9}$ The thyroid hormone levels can be variable, though most patients are euthyroid. ${ }^{1,8}$ Uncommonly, there can be reactive cervical adenopathy. ${ }^{5}$

The microscopic features are comparable with those seen in fibrosing diseases elsewhere in the body, leading to the suggestion that Reidel thyroiditis may be part of a systemic process and related to sclerosing cholangitis, mediastinal and retroperitoneal fibrosis, as well as orbital pseudotumor. ${ }^{1,4,13,14}$ At surgery, the thyroid gland is described as woody or stony hard and the thyroid is fixed to the adjacent neck structures. ${ }^{8,10}$ This usually limits resection to a debulking of the mass to relieve local symptoms. ${ }^{1,8,15}$ Medical treatment is usually with tamoxifen and/or high doses of steroids; however, such medical therapy has had variable results. ${ }^{1,5,9,15}$

Reidel thyroiditis demonstrates isointense-to-hypointense T1 signal intensity compared with adjacent normal muscle signal intensity and with even lower T2 signal intensity. This is in contrast to increased $\mathrm{T} 2$ hyperintensity usually seen in ma- 

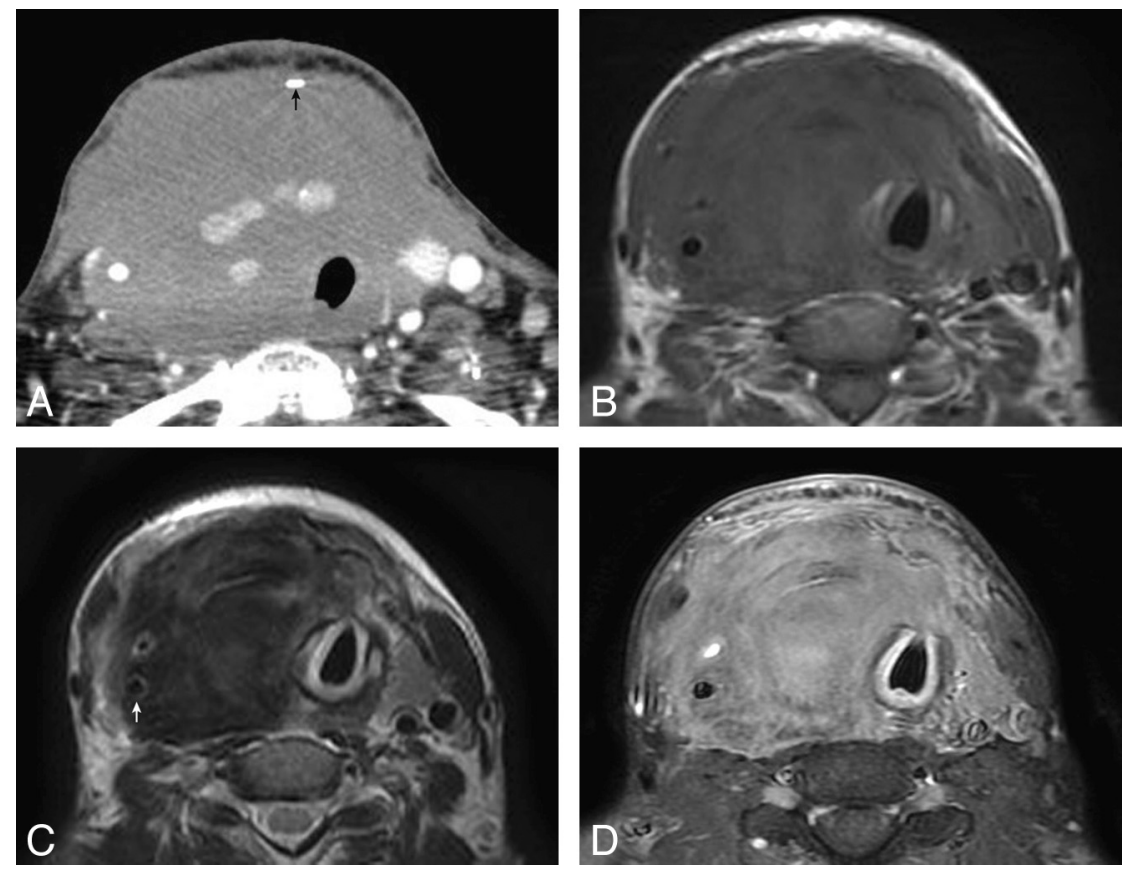

Fig 1. A, Axial contrast-enhanced CT scan shows an infiltrating mass replacing almost all of the thyroid gland. Islands of residual thyroid tissue are seen centrally and in the posterior left thyroid lobe. The fat planes between the mass and the trachea, esophagus, and flexor muscle of the cervical spine are infiltrated. A biopsy clip is seen anteriorly (black arrow). $B-D$, Axial T1-weighted $(B)$, T2-weighted $(C)$, and T1-weighted fat-suppressed contrast-enhanced $(D)$ MR images confirm the morphology of this highly infiltrating mass in the lower neck, replacing most of the thyroid gland. Overall the mass has a low T1-weighted and a lower T2-weighted signal intensity, and it enhances. The right internal carotid artery (white arrow) has a smaller caliber than the left artery.

lignancy. The pattern of enhancement is variable, ranging from homogeneous, such as in our case, to heterogeneous as reported in the literature. ${ }^{16}$ On CT, Reidel thyroiditis can be heterogeneous due to scattered islands of normal residual thyroid tissue in a surrounding bed of hypoattenuated fibrosis. Additionally, there is a disease entity that has been described in the literature that can mimic Reidel thyroiditis. This entity, also known as a paucicellular variant of anaplastic thyroid carcinoma, is a rapidly fatal disease with imaging characteristics and clinical presentation similar to those of Reidel thyroiditis. ${ }^{17}$ However, unlike Reidel thyroiditis, there may be necrosis within the lesion itself or within metastasized lymph nodes on imaging, infiltration of atypical spindle cells into the muscular layer of involved vessels, specific immunoreactivity staining, and a well-delineated margin between the anaplastic tumor and adjacent tissues. ${ }^{17}$

Because of its highly infiltrative nature, the imaging differential diagnosis includes aggressive thyroid tumors such as anaplastic carcinoma, squamous cell carcinoma, the tall cell variant of papillary thyroid carcinoma, and metastasis to the thyroid gland. ${ }^{8}$ Because virtually all cases of recurrent laryngeal nerve involvement by a thyroid tumor are secondary to a thyroid malignancy, the presence of a vocal cord paralysis additionally complicates the diagnosis. Last, the occasional associated cervical lymph adenopathy, when present, can further suggest a malignancy. The most reliable way to establish the diagnosis is via an open biopsy. ${ }^{8}$ Although rare, Reidel thyroiditis should be considered in the differential diagnosis of an infiltrative thyroid mass with an associated recurrent laryngeal nerve paralysis.

\section{References}

1. Vaidya B, Harris PE, Barrett P, et al. Corticosteroid therapy in Reidel's thyroiditis. Postgrad Med J 1997;73:817

2. Woolner LB, McConahey WM, Beahrs $\mathrm{OH}$. Invasive fibrous thyroiditis (Reidel's struma). J Clinl Endocrinol 1957;17:201-20

3. Mclver B, Fatourechi MM, Hay ID, et al. Graves' disease after unilateral Reidel's thyroiditis. J Clin Endocrinol Metab 2010;95:2525-26

4. Heufelder AE, Hay ID. Evidence of autoimmune mechanisms in the evolution of invasive fibrous thyroiditis (Reidel's struma). Clin Investig 1994;72:788-93

5. Ozbayrak M, Kantarci F, Olgun DC, et al. Reidel's thyroiditis associated with massive neck fibrosis. J Ultrasound Med 2009;28:267-71

6. Crile G Jr. Thyroiditis. Ann Surg 1948;127:640-54

7. LiVolsi VA. Pathology of the thyroid gland. In: Surgical Pathology of the Head and Neck, 2nd ed. New York: Marcel Dekker; 2001; 1680-81

8. Malotte MJ, Chonkich GD, Zuppan CW. Reidel's thyroiditis. Arch Otolaryngol Head Neck Surg 1991;117:214-17

9. Lo JC, Loh KC, Rubin AL, et al. Reidel's thyroiditis presenting with hypothyroidism and hypoparathyroidism: dramatic response to glucocorticoid and thyroxine therapy. Clin Endocrinol (Oxf) 1998;48:815-18

10. Vaidya B, Coulthard A, Goonetilleke A, et al. Cerebral venous sinus thrombosis: a late sequel of invasive fibrous thyroiditis. Thyroid 1998;8:787-90

11. Hoa SP, Chen JF, Yen KC. Reidel's thyroiditis associated with follicular carcinoma. Eur Arch Otorhinolaryngol 199:256:470-72

12. Rodriguez I, Ayala E, Caballero C, et al. Solitary fibrous tumor of the thyroid gland: report of seven cases. Am J Surg l Pathol 2001;25:1424-28

13. Yasmeen T, Khan S, Patel SG, et al. Reidel's thyroiditis: report of a case complicated by spontaneous hypoparathyroidism, recurrent laryngeal nerve injury and Horner's syndrome. J Clin Endocrinol Metab 2002;87:3543-47

14. Arnott EJ, Greaves DP. Orbital involvement in Reidel's thyroiditis. Br J Opththal $1965 ; 49: 1-5$

15. Few J, Thompson NW, Angelos P, et al. Reidel's thyroiditis: treatment with tamoxifen. Surgery 1996;120:993-99

16. Takahashi N, Kouichirou $\mathrm{O}$, Sakai $\mathrm{K}$, et al. MR findings with dynamic evaluation in Reidel's thyroiditis. J Clin Imaging 2002;26:89-91

17. Wan SK, Chan JK, Tang SK. Paucicellular variant of anaplastic thyroid carcinoma: a mimic of Reidel's thyroiditis. Am J Clin Pathol 1996;105: $388-93$ 